\title{
Statistical issues regarding the article by Liu et al.
}

\author{
Pedro Marques-Vidal (10 ${ }^{1}$
}

Received: 2 June 2020 / Revised: 17 July 2020 / Accepted: 13 August 2020 / Published online: 2 September 2020

(c) Springer Nature Limited 2020

\section{To the Editor:}

I read with interest the article by Liu et al. [1] on the effect of malnutrition status among elderly patients with COVID19. I have some questions regarding the analysis of the data.

- For each patient, four nutritional risk scores were computed. Comparing the prevalence of "at risk" patients between scores cannot be performed by simple chi-square, as the data come from the same sample (i.e., the data is not independent). In my opinion, a paired analysis should have been performed.

- Data from Table 5 is strange: an $\mathrm{OR}<1$ means that the variable is protective. Hence, an OR of 0.102 for NRS 2002 for LOS indicates that patients at risk using NRS 2002 have an almost tenfold LOWER likelihood of having a long LOS, which contradicts the statements in the text. It seems that the authors computed the OR for the non-at risk patients. Recomputing the OR leads to a value of 9.80 , which is more compatible with the effects of malnutrition on increased LOS.

- I am surprised that $p$ values of zero are presented. If a $p$ value is zero, then the corresponding test value is infinite.

\section{Compliance with ethical standards}

Conflict of interest The author declares no conflict of interest.

Publisher's note Springer Nature remains neutral with regard to jurisdictional claims in published maps and institutional affiliations.

\section{References}

1. Liu G, Zhang S, Mao Z, Wnag W. Clinical significance of nutritional risk screening for older adult patients with COVID-19. Eur J Clin Nutr. 2020; https://doi.org/10.1038/s41430-020-0659-7.
Pedro Marques-Vidal

Pedro-Manuel.Marques-Vidal@chuv.ch

1 Department of medicine, internal medicine, Lausanne University Hospital and University of Lausanne, 46 rue du Bugnon, 1011 Lausanne, Switzerland 